\title{
Optimal operation strategy for distributed battery aggregator providing energy and ancillary services
}

\author{
Kangning LIU ${ }^{1}$, Qixin CHEN ${ }^{1}$, Chongqing KANG ${ }^{1}$, Wei $\mathrm{SU}^{2}$, \\ Guobin ZHONG ${ }^{2}$
}

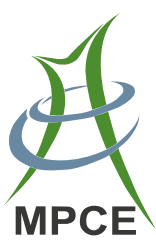

\begin{abstract}
The high penetration of distributed renewable energy raises a higher concern for the safe and economic operation of the smart grid. Distributed batteries equipped in demand-side can not only contribute to the reliability and security of the grid, but also make profits by participating in the electricity market, especially when distributed batteries are combined and operated by an aggregator. Considering the well-operated mechanism of performance based regulation (PBR) in the U.S. electricity market, it becomes increasingly lucrative for batteries to participate not only in energy markets for energy arbitrage, but also in ancillary service markets to provide regulation and peakload shaving services. In this study, distributed batteries are operated and coordinated by the aggregator, which simultaneously submits offers to the energy and the ancillary service markets as an individual entity. An optimal
\end{abstract}

CrossCheck date: 15 August 2017

Received: 13 September 2016/ Accepted: 16 August 2017 / Published online: 13 January 2018

(C) The Author(s) 2018. This article is an open access publication

$\triangle$ Qixin CHEN

qxchen@tsinghua.edu.cn

Kangning LIU

lknthu12@outlook.com

Chongqing KANG

cqkang@tsinghua.edu.cn

Wei SU

suwei@gddky.csg.cn

Guobin ZHONG

zhongguobin@gddky.csg.cn

1 Department of Electrical Engineering, Tsinghua University, Beijing 100084, China

2 Guangdong Electric Power Research Institute, Guangzhou 510620, China decision model is formulated for the aggregator to determine the operation and bidding strategy for the distributed batteries by considering the characteristics of batteries, including the terms of capacity, efficiency and degradation cost. Finally, a numerical case is conducted to evaluate the benefits of the decision model.

Keywords Distributed storage, Electricity market, Optimal operation, Ancillary service

\section{Introduction}

Storage is gaining increased importance with the high penetration of renewable energy in smart grid [1]. Significant research has been conducted in this context. For example, considering high-penetration wind and PV power, Lin Feng et al. evaluated the cost reduction for a hybrid energy storage system [2], and $\mathrm{Han} \mathrm{Li}$ et al. found an optimal energy management model for industrial microgrids, containing Li-ion batteries and super capacitors [3]. Storage can not only be integrated into the larger power grid by building centralized storage stations but can also be widely distributed on the demand side. With the continuing penetration of distributed renewable generation, distributed batteries equipped in the demand side are playing an increasingly critical role in addressing the urgent challenge of reliability and economy. For example, distributed batteries can smooth the distributed renewable generation and reduce the uncertainty [4]. Since distributed batteries equipped in the demand side are closer to consumers, they have more flexibility to provide reliable and secure consumer services.

In addition, there is ample opportunity for distributed batteries to achieve economic benefits by participating in 
the electricity market. With the opening of a new round of power system reforms in China, the electricity retail market will bring new business models and opportunities for the application of distributed batteries [5]. Although existing research on distributed batteries has usually concentrated on the aims of reducing cost and improving power quality, some studies have considered the scenarios of power market participation to maximize the profits. In [6], C. Goebel and D.S. Callaway investigated the market value of the electric car in California and concluded that utilizing those facilities in frequency regulation is a very promising business mode. The work in [7] establishes a new method to conduct an economic analysis of vehicle-to-grid (V2G) regulation services, mainly by simulating vehicle consumers' behaviors.

Distributed batteries are relatively small in capacity; thus, their individual participation in the electricity market is not economically feasible. In fact, because of the market access threshold, an individual distributed battery might have no access to provide grid services as bulk batteries do. To participate in the wholesale electricity market, they could be assembled as entities and operated by electricity retail aggregators, providing energy arbitrage, regulation services and peak-shaving services. Reference [8] analyzes the profitability of an aggregator business model in a microgrid using the so-called space exploration methodology. An optimal bidding strategy for a PHEVs aggregator is proposed in [9], mainly using stochastic optimization in the day-ahead electricity market. Pilot projects have also been conducted to exploit the economic potential of distributed batteries. The reflexe project [10], sponsored by the French Environment and Energy Agency (ADEME), for example, examines the aggregation flexibility to provide market service to the grid, mainly using the Zebra battery. In [11], the results from this project have been illustrated with a battery management system, and flexibility scheduling algorithms have been developed.

The income of batteries in the electricity market mainly consists of two parts: conducting energy arbitrage in the energy market and providing services in the ancillary service market.

Energy arbitrage is a classic application of batteries, due to the variability of electricity prices, and batteries can be engaged in so-called energy arbitrage [12, 13]. Reference [14] investigated the optimal operation of a micro-scale battery for energy arbitrage, which is a trade-off between the economic value and lifetime of the battery. However, many works have found this to not be economically feasible. After investigating the Danish power market, Ekman concluded that the revenue cannot compensate for the investment of storage [15]. Based on the entire life cycle of lithium-ion batteries, [16] assesses the economic potential of energy arbitrage in the Korean electricity market, drawing the conclusion that revenue is not sufficient to make up for the high cost at this stage in South Korea. It is also worth mentioning that the writer suggests that further financial gains may exist in the regulation market, ancillary services, and other applications.

Another major application for batteries is providing ancillary services, which compromise a wide range of offerings, such as synchronized reserve, non-synchronized service and regulation service. Among these, regulation service is especially lucrative. Compared with the traditional regulation resources, batteries have a higher ramp rate and can respond faster. However, because compensation is based on the committed capacity, regulation markets currently usually fail to consider the actual performance of regulation resources. To handle this problem, the FERC (Federal Energy Regulatory Commission) Order 755 [17] has developed pay-for-performance tariffs, which take both the speed and accuracy of providing frequency regulation into consideration, thus remedying the undue discrimination. This makes the regulation service increasingly lucrative for batteries. When batteries participate in the regulation market, they need to be engaged in the energy market so that they can meet the energy balance demand. Many scholars have conducted research on the joint optimization of the energy market and the regulation market, but fail to consider the performance based regulation (PBR) mechanism. J. Cho and A.N. Kleit developed a probability model to accommodate the PBR mechanism and calculated the net present value of many years' income based on the discount rate [18]. G. He, Q. Chen, C. Kang et al. proposed a battery storage optimal bidding strategy in the joint day-ahead market, with the consideration of the PBR mechanism and battery cycle life model, which makes the economic evaluation of storage more accurate [19].

Unlike other types of energy storage, battery lifetimes are usually more sensitive to charging and discharging processes during the electricity market participation. Discharging or charging too fast will heavily influence the battery lifetime. Therefore, it is vital to balance the aggressive revenue-oriented behavior and the conservative tendency to prolong battery lifetime [14]. Thus, a deep investigation of the battery characteristics and building an appropriate battery model are crucial for profit optimization. Battery models mainly contain two types: the physical model and the mathematical model. Relying on the methodology of Model Predictive Control (MPC), M. Koller et al. defined a degradation cost function for the optimal control of batteries [20]. Reference [21] shows that lithium-ion battery life cycle loss changes little with different DOD (Depth of Discharge), and thus adopted a linear simplified model. This model is very practical and qualifies the precision standard for cost modeling based on market service provisions. 
Moreover, most of the works above are concerned with bulk batteries. Some discuss the market participation of distributed batteries based on the assumption that all the batteries share the same characteristics. However, this assumption is mismatched from actual circumstances: distributed batteries often contain equipment in different conditions, with different technical characteristics. The current study investigates different types of batteries and chooses the lithium-ion battery as the desirable distributed battery. Characteristic differences among these specific batteries are included in the analysis. In addition, this study considers the market participations of the distributed battery aggregators in the wholesale energy market and the regulation market with the PBR mechanism. Different market price scenarios are also taken into account to stabilize the profits.

The contributions of this study are as follows:

1) An optimization model is proposed, achieving the optimal operation and bidding of distributed battery aggregators.

2) The degradation cost function for the distributed lithium-ion battery is suggested. This function combines lithium-ion battery characteristics (capacity, efficiency, degradation loss) with specific work conditions.

3) Based on this model, a numerical case is used as an example to evaluate the profits with and without aggregator operation.

The rest of this article is organized as follows. Section 2 describes the business mode of distributed battery market participation. Section 3 investigates different types of batteries and builds the battery model for the chosen distributed battery, which is lithium-ion. Section 4 presents the optimal decision model for the distributed battery aggregator. The case study results are discussed in Section 5, and conclusions are drawn in Section 6.

\section{Business mode for distributed battery aggregator}

\subsection{Electricity market participation}

This study focuses on electricity market revenues from two sources: the energy market and the regulation service market. The electricity market mechanism in the current study is similar to that in the PJM. Considering that the capacity of the distributed battery is much smaller than the traditional unit, we assume that a distributed battery has little effect on the entire electricity market and adopt the price-taker assumption. By participating in the energy market, a distributed battery can gain profits through energy arbitrage, which is a strategy of buying at low power price while selling at a high power price $[12,13]$. The regulation market considered in this study refers to that in PJM, which is circumstantiated in [17]. It mainly contains two types of signals: the traditional regulation signal $(\operatorname{Reg} \mathrm{A})$ and the dynamic regulation signal (RegD). The former is used for traditional resources with a limited ramp rate, while the latter is used for fast-responding resources. In addition, the payments are capacity-based to compensate for reservation cost and mileage-based to compensate for costs related to the movement of the resource.

\subsection{Control mechanisms of distributed battery aggregator}

The proposed business mode assumes that distributed batteries are owned by consumers. Because accurate price information cannot be accessed in advance, it is necessary for aggregators to predict the future electricity price before bidding.

The aggregator's responsibility mainly consists of two parts: 1) participating in the day-ahead wholesale market using optimal bidding strategies; and 2) distributing the specific biddings among distributed batteries. On one hand, the aggregator needs to satisfy the consumers' own service needs and improve the power quality; on the other hand, it signs contracts with consumers that enable them to utilize batteries, resulting in achievement of its own profit optimization. More than that, in the process of shaping the demand-side strategies, the aggregator needs to fully consider the specific reliability requirement of consumers, from which the aggregator decides the approach to participate in the electricity market.

The control structure we consider is shown in Fig. 1 [22]. The distributed battery is controlled by the Individual Battery Control Unit, which also contributes to stabilize the secondary voltage and service load. The Control Center in the Distribution Network gathers information from the

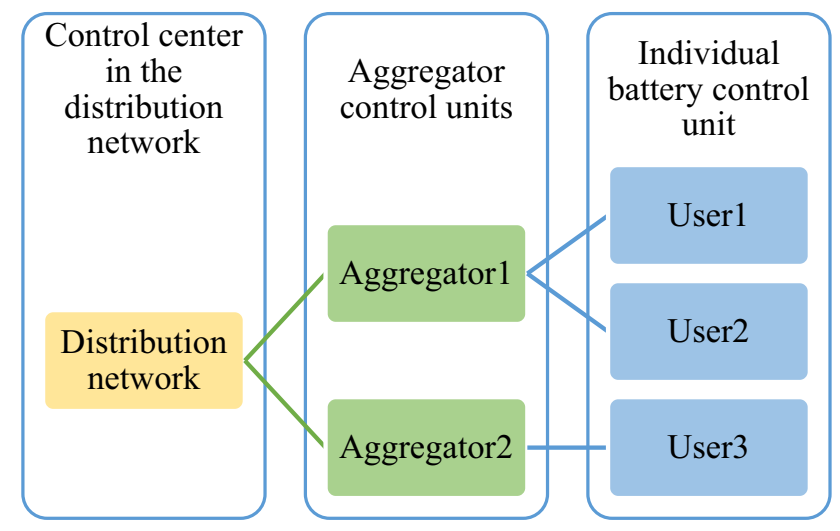

Fig. 1 Control mechanism of the aggregator's agency operation 
Aggregator Control Units. The Individual Battery Control Unit receives instruction from the Aggregator Control Units about the time and quantity that the individual battery exchanges power with the grid, which it then implements. There is no direct information exchange within different aggregators, yet the Control Center in the Distribution Network could make the indirect information exchange possible if necessary, which can be achieved through gathering information from one aggregator and sharing it with another aggregator.

\section{Life model of desirable distributed battery}

\subsection{Economic comparison among different batteries}

Based on the fact that lithium-ion batteries are widely distributed on the demand side and their characteristics well qualify the requirements of the market participation, lithium-ion batteries are chosen as desirable distributed batteries with which to perform the analysis in this study.

Considering the large number of commercially accessible battery technologies and the complexity of different applications, the selection of the most suitable battery for the regulation service is a complex process. In addition, there is limited number of previous studies that has been conducted on battery selection.

Although various kinds of distributed batteries are qualified for market participation, they vary in capacity, power, response time, cycle life loss, cost, and energy efficiency. In addition, during different service provisions and application scenarios, technical requirements can be different.

Reference [23] proposes a general methodology to choose the most suitable energy storage for a specific service. Combined with this general approach, for applications of the regulation service, it is necessary to have high power capacity, small life loss during cycles with shallow depth of discharge (DOD), fast response characteristics and the ability of longer-term storage.

Figures 2 and 3 compare the differences between battery types in cycle life and energy efficiency, respectively. Generally, cycle life indicates the number of charge/ recharge cycles before a battery's performance starts to visibly reduce.

These figures show that the lithium-ion battery has the highest energy efficiency and the highest cycle life when the DOD is shallow. In [23], the authors also compare the different battery cycle costs with specific DOD. It concludes that when the cycle is shallow, the lithium-ion battery has the lowest cycle cost.

In addition, the lithium-ion battery also has a low selfdischarge rate (approximately 1\%-3\% per month), which

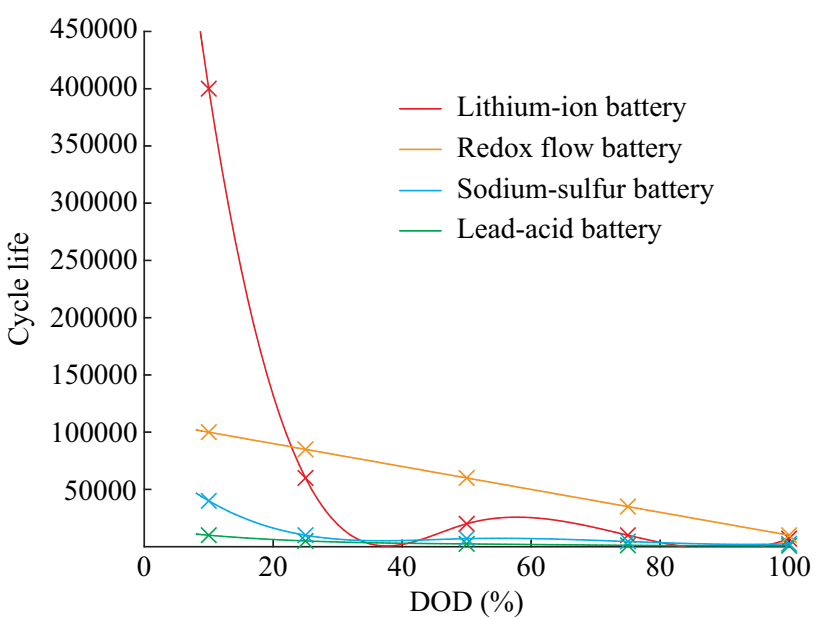

Fig. 2 Life cycle for different kinds of batteries for different DODs

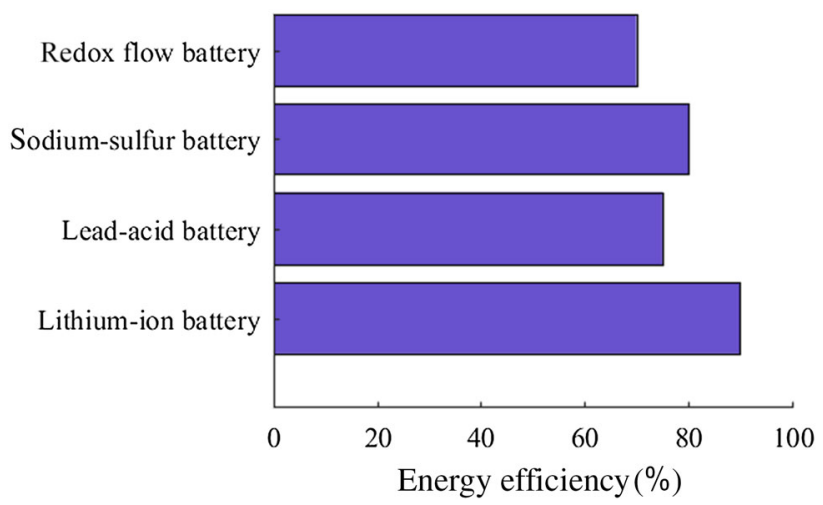

Fig. 3 Energy efficiency for different kinds of batteries

makes it more appealing in comparison with other batteries. In addition, lithium-ion batteries are very flexible in capacity and size and have already been widely used in the distribution network, such as in electric vehicles. Thus, the lithium-ion battery is chosen for demand-side usage in this study, which requires frequent responses with a small amount of energy and long duration.

\subsection{Life model of the lithium-ion battery}

There are many works investigating the cycle models of lithium-ion batteries, and these mainly contain two types: physical models and mathematical models. However, because of the uniqueness of each battery, a single model cannot perfectly describe the battery characteristics. Having considered the availability of battery parameters and the convenience of the calculation process and model build-up, it is practical to adopt mathematical models.

Reference [24] models the calendar life and the cycle life of lithium-ion batteries. It finds that time and temperature strongly impact the calendar life and that calendar 
loss follows the square root of time. The calendar life of a lithium-ion battery can be modeled as follows:

$q_{\text {loss }} \%=14876 \exp (-24.5 \mathrm{~kJ} / R T) t_{\text {days }}^{0.5}$

where $q_{\text {loss }} \%$ is the percentage of capacity loss; $R$ is the gas constant; $T$ is the absolute temperature; $t_{\text {days }}$ is the number of days in the operation.

Calendar life performance is a direct indication of irreversible self-discharge loss. According to previous reviews, the lithium-ion battery self-discharge loss is small. As a result, we focus more on cycle loss in this study.

Repeated deep cycling damages the battery lifetime because it results in a gradual reduction in its capacity [25]. In the current study, experimental life-cycle tests were conducted to record data. A model to compute the incremental impact of one cycle of a specific DOD is illustrated as follows:

$c_{\text {Li-ion }}=20 /\left(33000 \mathrm{e}^{-0.06576 N_{\text {DoDcyc }}}+3277\right)$

Battery cycles are normally quite shallow when providing regulation services. Therefore, it is reasonable to adopt a linear model; we choose the model in [21] for the convenience of parameter availability. Research finds that the lithium-ion battery is sensitive to the equivalent cycle time rather than the DOD. The cycle degradation cost can be expressed as:

$C^{x}=|k / 100| \cdot x / B \cdot C^{B}$

where $k$ denotes the approximate linear slope of the battery life loss; $B$ denotes the battery capacity; $C^{B}$ denotes the battery cost, and $x$ denotes the accumulated energy charged and discharged from the battery.

\section{Optimal model for distributed battery aggregator}

In this section, the bidding and distribution model in a joint electricity market is presented in detail. The decision variables are $p_{t, v}^{e, d s g}, p_{t, v}^{e, c h g}$ and $p_{t, v}^{r e g}, p_{t, v}^{e, d s g}$ and $p_{t, v}^{e, c h g}$ denotes the power discharge and charge in the energy market by battery $v$ at time $t . p_{t, v}^{r e g}$ denotes the bidding in the regulation market by battery $v$ at time $t$. By optimizing the three variables, the aggregator achieves its maximum profits.

\subsection{Objective function}

The objective function aims to achieve maximum profits in a given day, as described in (4). The total profits mainly consist of the revenues and the costs. This function is a weighted average of profits in different market price scenarios, which may have different probabilities of occurrence. In this way, the aggregator can predict a series of market price scenarios in advance and choose different probabilities of occurrence using their current data. This contributes to stabilize the aggregator's profits. $\gamma_{s}$ denotes the probability of price scenario $s$. This factor may be determined by the subjective preference (e.g., the specific price scenario that the aggregator mainly focuses on), the objective prediction (e.g., forecast through systematic analysis of historical data), or both. $r_{s, t}^{e m}$ and $r_{s, t}^{r e g}$ denote revenue from the energy market and revenue from the regulation market in scenario $s$ at hour $t$, respectively. $c_{t}^{o p}$ denotes the operation cost at hour $t, c^{\text {deg }}$ denotes the total battery degradation cost, and $c^{m}$ denotes the maintenance cost.

$$
\begin{aligned}
& p_{\text {totalprofits }} \\
& \quad=\max \sum_{s \in S} \gamma_{s} \sum_{t \in H}\left(r_{s, t}^{e m}+r_{s, t}^{r e g}\right)-\sum_{t \in H} c_{t}^{o p}-c^{d e g}-c^{m}
\end{aligned}
$$

\subsection{Constraints: revenues and costs}

For the revenues, the energy market revenue and the regulation market revenue are derived in (5) and (6), respectively. $\lambda_{s, t}^{e}$ denotes the energy price at hour $t$ in scenario $s, b_{t}^{e}$ is the aggregator's total energy market bidding at hour $t$, which will be distributed and broken down to specific energy biddings among the distributed batteries, $\Delta t$ is the time interval between time $t$ and time $t+1$, usually one hour, $r_{s, t}^{\text {reg,cap }}$ denotes the capacity payment, and $r_{s, t}^{\text {reg,perf }}$ denotes the mileage payment.

The regulation market revenues consist of the capacity and mileage payments, determined in (7) and (8), respectively. $\lambda_{s, t}^{\text {reg,cap }}$ and $\lambda_{s, t}^{\text {reg,perf }}$ denote the regulation capacity price and regulation performance price at hour $t$, respectively, $b_{t}^{\text {reg }}$ denotes the aggregator's total regulation market bidding at time $t$, and $S^{\text {perfscore }}$ denotes the regulation performance score of the battery in providing a PBR service. Because batteries virtually share the characteristics of fast response and high accuracy, the $S^{\text {perfscore }}$ is considered the same for all batteries and is constant through different time periods. $R_{s, t}^{\text {mileage }}$ is the mileage ratio for regulation resource at time $t$ in scenario $s$.

$r_{s, t}^{e m}=\lambda_{s, t}^{e} b_{t}^{e} \Delta t$

$r_{s, t}^{r e g}=r_{s, t}^{r e g, c a p}+r_{s, t}^{r e g, p e r f}$

$r_{s, t}^{\text {reg,cap }}=\lambda_{s, t}^{\text {reg,cap }} b_{t}^{\text {reg }} S^{\text {perfscore }}$

$r_{s, t}^{\text {reg,perf }}=\lambda_{s, t}^{\text {reg,perf }} b_{t}^{\text {reg }} R_{s, t}^{\text {mileage }} S^{\text {perfscore }}$

On the cost side, the operation $\operatorname{cost} c_{t}^{o p}$ is proportional to the amount of power, shown in (9). $c_{o p}$ denotes the 
operational cost per unit energy, $b_{t}^{e, s e l l}$ and $b_{t}^{e, b u y}$ denote the total energy bidding sold to the grid and bought from grid, respectively, and $\beta_{t}$ indicates the average energy consumed in the regulation (up or down) in time period $t$ for a $1-\mathrm{kW}$ regulation bidding, which is determined by the generated RegD signal.

The battery degradation cost is closely related to the battery characteristics. The relationship between individual degradation costs and the total degradation cost is shown in (10). The $c^{\text {deg }}$ denotes the total battery degradation cost, and $c_{v}^{d e g}$ denotes the degradation cost of battery $v$.

Based on (3) from [21], the linear degradation cost model is reasonably adopted. After considering the specific application scenario, the degradation cost model is built in (11). The $p_{t, v}^{e, d s g}$ and $p_{t, v}^{e, c h g}$ denote the actual amount of power that battery $v$ discharged and charged, respectively, in the energy market, and $\beta_{t} p_{t, v}^{\text {reg }} / \eta_{v}^{d s g}$ and $\beta_{t} p_{t, v}^{\text {reg }} \eta_{v}^{\text {chg }}$ denote the power of battery $v$ discharged during regulation up in hour $t$ and the power of battery $v$ charged during regulation down in hour $t$, respectively. Together, they constitute the total energy that battery $v$ has charged and discharged during time period $t$. Their sum over all time periods is proportional to the degradation cost. $C_{v}^{\text {deg }}$ denotes the cost of battery $v, M_{v}$ denotes the degradation slope of battery $v$, and $\eta_{v}^{\text {chg }}$ and $\eta_{v}^{d s g}$ denote the charging and discharging efficiency of battery $v$, respectively.

In addition, the maintenance cost represented in (12) is proportional to the maximum power the batteries can provide. $c^{m}$ denotes the maintenance cost, $\lambda_{m}^{e}$ denotes the operational cost per unit energy, and $P_{\max }$ denotes the total maximum power of all distributed batteries.

$$
\begin{aligned}
c_{t}^{o p}= & c_{o p}\left[\left(b_{t}^{e, s e l l}+b_{t}^{e, b u y}\right) \Delta t+2 \beta_{t} b_{t}^{\text {reg }}\right] \\
c^{\text {deg }}= & \sum_{v \in V} c_{v}^{d e g} \\
c_{v}^{\text {deg }}= & C_{v}^{b a t}\left|\frac{M_{v}}{100}\right| \\
& {\left[\sum_{(t \in T)}\left(\begin{array}{l}
p_{t, v}^{e, d s g} \Delta t+p_{t, v}^{e, c h g} \Delta t+ \\
\beta_{t} p_{t, v}^{r e g} / \eta_{v}^{d s g}+\beta_{t} p_{t, v}^{\text {reg }} \eta_{v}^{\text {chg }}
\end{array}\right)\right] / E_{v, \text { max }} }
\end{aligned}
$$

$c^{m}=\lambda_{m}^{e} P_{\max }$

\subsection{Constraints: biddings and demand-side distributions}

The total energy bidding is determined by the energy sold and energy bought. This relationship is illustrated in (13). A certain battery $v$ at a specific time period $t$ cannot be both charged and discharged simultaneously, which means that $p_{t, v}^{e, d s g}$ and $p_{t, v}^{e, c h g}$ could not both be positive. However, since there are many distributed batteries, it is technically possible for the aggregator to sell and buy at the same time. To be specific, at time period $t$, some batteries are chosen to be charged, whereas others are chosen to discharged. Nevertheless, out of economic consideration, the aggregator normally either sells or buys at a specific time period. Optimally, the total energy bidding should be distributed among the batteries, which is represented in (14) and (15). Similarly, the total regulation market bidding is distributed as in (16). The total maximum power is the sum of individual battery's rated power, as in (17), where $P_{v, \text { max }}$ denotes the rated power capacity of battery $v$.

$b_{t}^{e}=b_{t}^{e, s e l l}-b_{t}^{e, b u y}$

$b_{t}^{e, s e l l}=\sum_{v \in V} \eta_{v}^{d s g} p_{t, v}^{e, d s g}$

$b_{t}^{e, b u y}=\sum_{v \in V} p_{t, v}^{e, c h g} / \eta_{v}^{c h g}$

$b_{t}^{r e g}=\sum_{v \in V} p_{t, v}^{r e g}$

$P_{\max }=\sum_{v \in V} P_{v, \max }$

\subsection{Constraints: battery's characteristics}

The change in the state of charge (SOC) is determined by the effects of the charging and discharging processes in the energy market and the regulation market and is determined in (18). The $S O C_{t, v}$ denotes the state of charge of battery $v$ in hour $t, \alpha$ denotes the self-discharging rate of battery $v$ between $t$ and $t+1$, which is normally sufficiently small to be negligible, and $E_{v, \max }$ denotes the rated energy capacity of battery $v$. The discharging and charging process in the energy market will influence the SOC of the battery. In addition, despite the energy-neutral characteristic of the RegD signal, energy loss occurs when providing regulation services consisting of charging and discharging. The $\beta_{t} p_{t, v}^{r e g} \eta_{v}^{\text {chg }}$ and $\beta_{t} p_{t, v}^{\text {reg }} / \eta_{v}^{d s g}$ represent the energy charged to the battery during regulation down and discharged from the battery during regulation up in time period $t$, respectively.

$$
\begin{aligned}
& S O C_{t, v}=(1-\alpha) S O C_{t-1, v}+\left(p_{t, v}^{e, c h g} \Delta t-p_{t, v}^{e, d s g} \Delta t\right. \\
& \left.+\beta_{t} p_{t, v}^{r e g} \eta_{v}^{c h g}-\beta_{t} p_{t, v}^{r e g} / \eta_{v}^{d s g}\right) / E_{v, \max } \\
& \quad \forall t \in T, v \in V
\end{aligned}
$$

The maximum and minimum SOC of every battery is limited by (19) to protect the battery. The $S_{O C} C_{v}^{\min }$ and $S O C_{v}^{\max }$ denote the minimum value and maximum value of the SOC, respectively. 
$S O C_{v}^{\min } \leq S O C_{t, v} \leq S O C_{v}^{\max } \quad \forall t \in T, v \in V$

Considering the energy constraints for energy market bidding and power reserve requirements for the regulation market and consumers, the aggregator needs to meet certain requirements, as shown in (21). This means that a battery must be able to maintain the fully deployed output level for at least $h^{\text {reg }}$ (typically $15 \mathrm{~min}$ ) time for the regulation service and for at least $h^{\text {consumer }}$ to provide $p_{t, v}^{\text {consumer }}$ power reserved for the consumer. For a specific time $t$ and after the charging process, the SOC could not exceed the maximum level, whereas after the discharging process, the SOC needs to be larger than the minimum level. Therefore, for the $\left(p_{t, v}^{e, c h g}-p_{t, v}^{e, d s g}\right) \Delta t$, the energy bidding must be taken into account.

$$
\begin{aligned}
& \sum_{v \in V}\left[S O C_{v}^{\min } \cdot E_{v, \max }+\left(p_{t, v}^{\text {reg }} h^{\text {reg }}+p_{t, v}^{\text {consumer }} h^{\text {consumer }}\right) / \eta_{v}^{d s g}\right. \\
& \left.+\left(p_{t, v}^{e, d s g}-p_{t, v}^{e, c h g}\right) \Delta t\right] \\
& \leq \sum_{v \in V}\left(\operatorname{SOC}_{t, v} \cdot E_{v, \max }\right) \\
& \left.\left.\leq \sum_{v \in V} \begin{array}{c}
{\left[S O C_{v}^{\max } \cdot E_{v, \max }+\left(p_{t, v}^{e, d s g}-p_{t, v}^{e, c h g}\right) \Delta t+\right.} \\
\text { reg }
\end{array} h^{\text {reg }}-p_{t, v}^{\text {consumer }} h^{\text {consumer }}\right) \eta_{v}^{\text {chg }}\right]
\end{aligned}
$$

The charging and discharging rate of every battery must be under the maximum charging and discharging rate because of its characteristics, as in (22) and (23). The biddings must be within the upper and lower limits; thus, batteries should reserve a capacity of $\sigma$ units for the regulation service, as in (24) and (25).

$0 \leq p_{t, v}^{e, d s g} \leq P_{v, \max }$

$0 \leq p_{t, v}^{e, c h g} \leq P_{v, \max }$

$b_{t}^{e}-\sigma b_{t}^{r e g} \geq-P_{\max }$

$b_{t}^{e}+\sigma b_{t}^{r e g} \leq P_{\max }$

\section{Case study}

Based on the model built above, we conducted the following case study using GAMS, on a PC with an Intel Core $7 \mathrm{CPU}(2.4 \mathrm{GHz})$ and $8.0 \mathrm{~GB}$ RAM. A brief introduction of GAMS can be found in [26].

In the case study, two scenarios are compared to determine the economic differences:

1) An aggregator operates ten distributed batteries to participate in the electricity market (both the energy market and the regulation market);

2) The same ten distributed batteries are used, but every consumer operates its battery individually. In this case, an individual battery is too small to meet market requirements to provide grid service and could only gain benefits through peak and off-peak power price difference, which means it has no access to participate in the regulation market. The model used in this scenario is very similar to the model in Section 4. The differences mainly exist in these aspects: a) the individual battery could not gain regulation market revenue, and regulation market constraints are thus discarded; b) the object function is the profit of every individual battery, not the total profits of all distributed batteries.

\subsection{Basic data}

The 2014 PJM historical price data are used for this study. We selected market prices of one month to represent thirty scenario market prices needed for the case study, including the day-ahead electricity price and regulation market price (capacity price and mileage price).

The average scenario market prices are drawn in Fig. 4; the average scenario market prices are indications for the predicted prices to some extent, based on which aggregator conducts the optimization.

Figure 4 shows significant variations in the energy price and the capacity price, while the mileage price is sustained at a low level. The variations in the energy price, therefore, create the opportunity for batteries to perform energy arbitrage.

To compare the results, the two situations are based on the same group of ten distributed batteries. To compare the degradation differences, the ten distributed batteries are distinct from each other in their degradation slopes, as in Fig. 5. For the convenience of comparison, the degradation slopes are given regularly. The realistic value can be obtained from the specific battery's production sheet. For

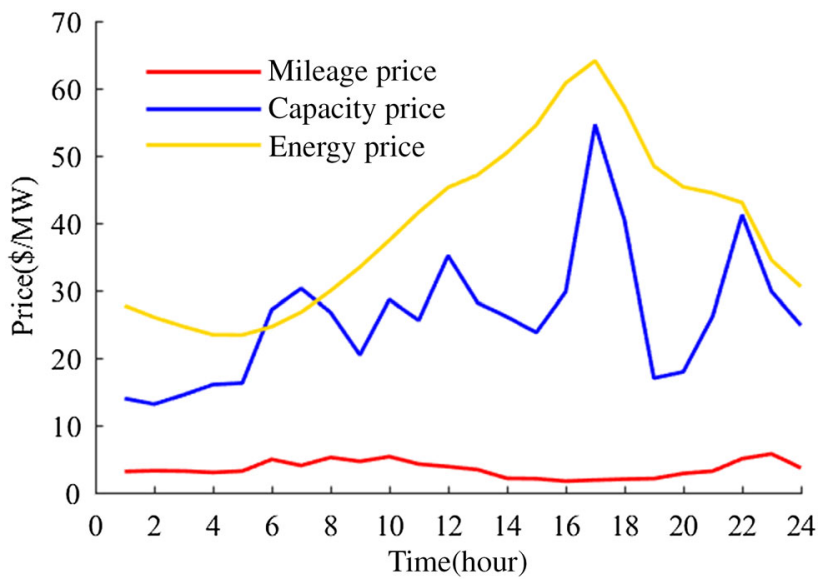

Fig. 4 Scenario market prices 


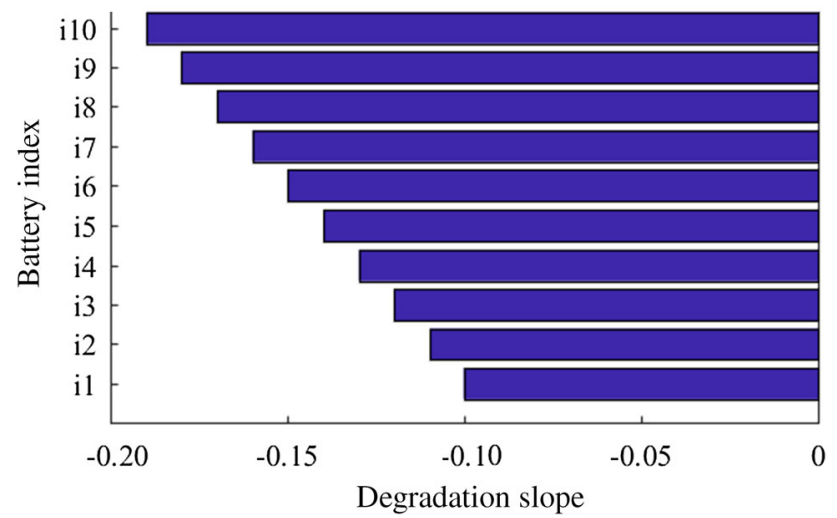

Fig. 5 Degradation slopes of different distributed batteries

example, the characteristic of the A123 - 'AMP20 Lithium Ion Prismatic Cell' can be found in [27]. The degradation slope is a parameter used to indicate the cost of the battery degradation per unit power during charging or discharging. Given that the other conditions are the same, the degradation cost increases with the absolute value of the degradation slope.

With this one exception, the ten batteries are considered to share the same capacity, energy efficiency and other parameters, as in Table 1 . The actual parameters of the lithium-ion battery obtained from different sources may vary. Therefore, in this study, the battery cost is considered to be $500 \$ / \mathrm{kW}$ [28], and the operation and maintenance cost is based on [28] with some minor adjustments, the capacity unit is set in $\mathrm{kW}$ for the convenience of calculation, and the capacity unit of the distributed lithium-ion battery is in $\mathrm{kW}$.

The initial SOCs of all the distributed batteries are set to their technically minimum levels; in this case, they are assumed to be zero. The SOC levels may vary in the process of market participation. However, by the end of the operation time, all return to their minimum levels within the constraints. Since the objective function is the maximum profit in a day, batteries will release their stored energy as much as possible.

Table 1 Parameters of lithium-ion batteries

\begin{tabular}{ll}
\hline Parameter & Value \\
\hline Operation cost $(\$ / \mathrm{MWh})$ & 3.76 \\
Maintaining cost $(\$ / \mathrm{MW})$ & 12.7 \\
Energy efficiency & 0.95 \\
Battery cost $(\$ / \mathrm{kW})$ & 500 \\
Performance score & 0.9 \\
Mileage ratio & 3 \\
Capacity $(\mathrm{kW})$ & 40 \\
Energy capacity $(\mathrm{kWh})$ & 40 \\
\hline
\end{tabular}

In the current study, $p_{t, v}^{\text {consumer }}$ is assumed to be zero. A detailed discussion on consumer behavior is beyond the scope of the present study.

\subsection{Results: biddings and economic outcomes}

The bidding results of Scenario 1 are drawn in Fig. 6, in which the columns denote the aggregator's total biddings in the regulation market and the energy market, respectively. The lines indicate different market prices over 24 hours. This shows that batteries are mainly engaged in the regulation market, only buying power from the grid at a low price to make up for the energy loss during the regulation service provision. Distributed batteries mainly conduct energy biddings at hours 1-4. In addition, because of the total constraints of the battery capacity characteristics, shown in (24) and (25), the energy biddings limit the regulation biddings to a certain extent. Except for hours $1-4$, in which the energy market biddings are primarily concentrated, all the distributed batteries bid to their fullest extent in the regulation market at all other hours. This outcome is reasonable since the regulation market is lucrative. Additionally, since the $\operatorname{RegD}$ signal shares energy-neutral characteristics, full biddings in the regulation market are within the constraints.

The energy market bidding amount drawn in Fig. 7 shows the aggregator's specific distribution of the energy market bidding among the distributed batteries. Combined with information from Fig. 5, it is apparent that batteries i1 and i2 (with the smallest degradation) are utilized with the highest priority. Then, battery i3 and battery i4 are used in sequence. In this way, the aggregator can minimize the degradation cost in the energy market participation.

Figure 8 is shown to investigate the distribution of the regulation market biddings. Combined with information

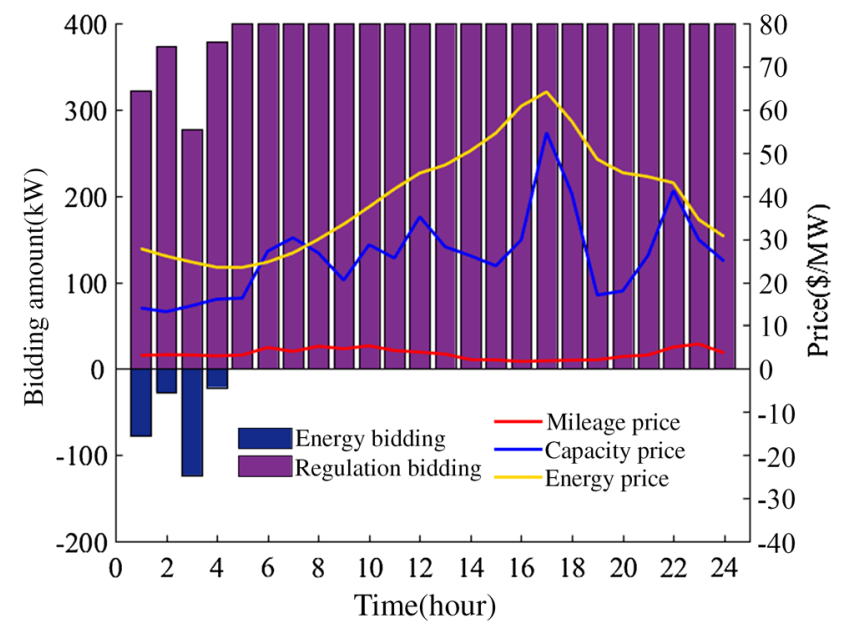

Fig. 6 Aggregator's bidding results from Scenario 1 


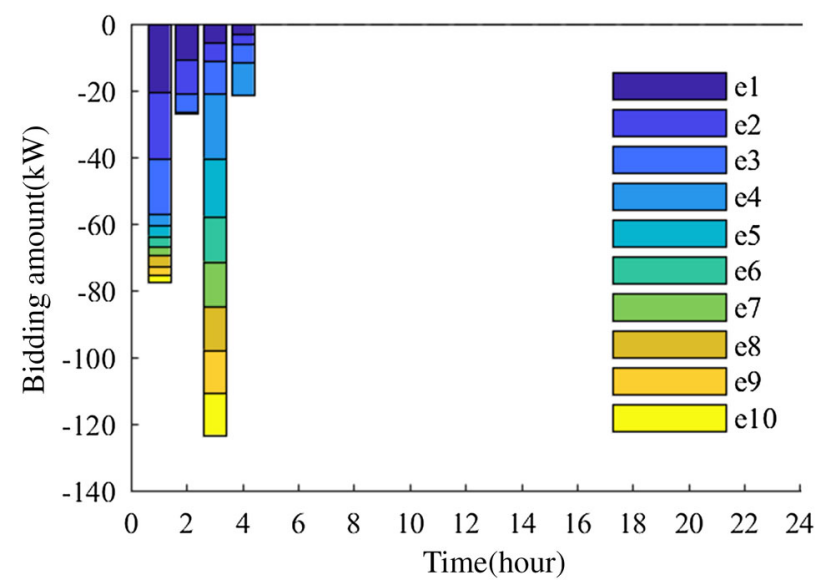

Fig. 7 Distributed battery bidding in energy market

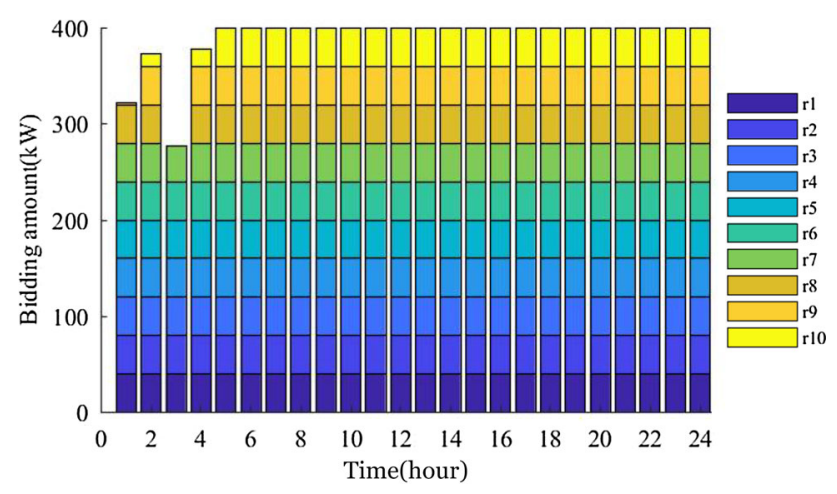

Fig. 8 Distributed battery bidding in regulation market

from Fig. 5, it can be seen that batteries with high degradation cost (such as batteries i8, i9 and i10) do not bid at their maximum capacities from hours $1-4$. This is because there must be compensation for the reservation capacity occupied by the energy biddings. The aggregator chooses the batteries with high degradation cost for reservation, while it chooses the comparatively inexpensive batteries to participate in the regulation market. Briefly, the distributed batteries are different in degradation characteristics, based on which optimal strategy the aggregator uses to determine priorities during bidding distributions.

The bidding results of Scenario 2 are given in Fig. 9. In this figure, columns i1-i10 denote biddings in the energy market. Figure 9 shows that batteries are only discharged when the power price is high and are charged when the power price is low. In addition, batteries with low degradation cost (such as battery i1) bid more in the energy market through a larger time range, which indicates that profit is closely related to battery degradation cost.

Comparing Figs. 6 and 9, it can be concluded that if the batteries are operated by consumers themselves, the battery

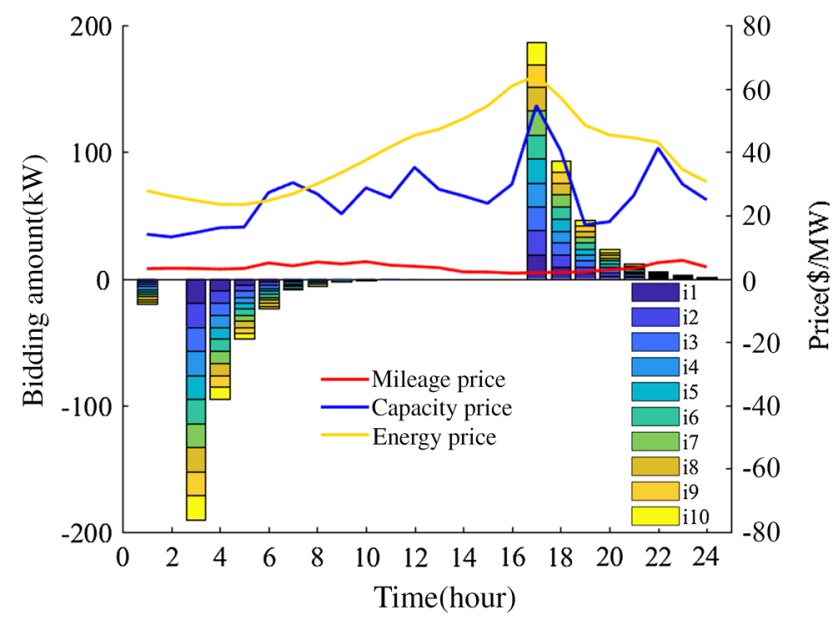

Fig. 9 Consumers' bidding results of Scenario 2

utilization rate is relatively low. However, when batteries are operated by the aggregator for joint optimizing between the energy market and the regulation market, battery resources can be used more effectively.

The economic results are compared in Table 2. In Scenario 2, compared to the relatively small energy market revenue, the degradation cost of the battery and the maintenance cost given in this condition are combined, resulting in a relatively higher amount. As a consequence, the revenue is insufficient to provide financial gains when batteries only gain benefits through peak and off-peak power price differences. This outcome corresponds to the conclusion drawn in [15] and [16].

In contrast, when batteries are operated by the aggregator in Scenario 1, the profit is considerable. When the revenue in the energy market is negative, the main source of revenue is the participation in the regulation market. Although the capacity revenue is higher than the mileage revenue, the latter does occupy a considerable part of the profits. Without the mileage revenue brought by the PBR mechanism, the aggregator's total profits will decrease by $25.17 \%$. The greatest portion of the total costs consists of

Table 2 Economic comparison

\begin{tabular}{llc}
\hline Parameter $(\$)$ & Scenario 1 & Scenario 2 \\
\hline Total profit & 304.88 & -1.99 \\
$\begin{array}{l}\text { Energy market } \\
\text { revenue }\end{array}$ & -6.69 & 9.03 \\
$\begin{array}{l}\text { Regulation market } \\
\quad \text { revenue }\end{array}$ & $\begin{array}{c}339.49 \text { (capacity part: 262.75; } \\
\text { mileage part: 76.74) }\end{array}$ & 0.00 \\
Operation cost & 0.83 & 0.38 \\
$\begin{array}{l}\text { Degradation cost } \\
\text { Maintaining cost }\end{array}$ & 21.98 & 5.54 \\
\hline
\end{tabular}


battery degradation costs. Therefore, the aggregator focuses most on degradation costs to reduce the costs.

Without loss of generality, we may well conclude that the aggregator, which possesses the ability to access the wholesale electricity market and conduct optimization based on the distributed battery characteristics, can flexibly allocate the biddings among different batteries. As a result, it is more economically feasible to operate distributed batteries than consumer-based ones. In addition, revenues from the regulation market play a more vital role in the total profits than revenues from simply conducting energy arbitrary. Moreover, compared to the regulation market without such a mechanism, the PBR mechanism enables batteries to gain a considerable amount of additional profit.

\section{Conclusion}

It is becoming increasingly lucrative for batteries to participate in the electricity market, especially the regulation market, with the PBR mechanism. In this study, a profit-oriented business mode is introduced with the consideration of actual constraints. In this model, an optimal strategy for the aggregator, which combines the actual market information with lithium-ion battery characteristics, is presented. Scenario market prices are also taken into account to stabilize the profits.

A case study was conducted to determine the benefits of aggregator operation. By making full use of batteries distributed on the demand side, the business mode that the aggregators operate and coordinate with distributed batteries is economically superior to individual consumer operation. This is mainly due to two reasons: 1) the aggregation mode enables the relatively small distributed batteries to participate in the regulation market, which sets certain requirements that are unattainable for individual batteries; and 2) the aggregator is able to make the best use of information and approach the optimal bidding and operation strategy to maximize profits. It is thus reasonable to conclude that the model in which distributed batteries are operated and coordinated by the aggregator is technically and economically feasible.

Acknowledgements This work was supported by Major International (Regional) Joint Research Project of National Natural Science Foundation of China (No. 51620105007), and Scientific Research Project of Guangdong Electric Power Research Institute.

Open Access This article is distributed under the terms of the Creative Commons Attribution 4.0 International License (http:// creativecommons.org/licenses/by/4.0/), which permits unrestricted use, distribution, and reproduction in any medium, provided you give appropriate credit to the original author(s) and the source, provide a link to the Creative Commons license, and indicate if changes were made.

\section{References}

[1] Albuyeh AIAF (2009) Grid of the future. IEEE Power Energy Mag 7(2):52-62

[2] Feng L, Zhang JN, Li GJ et al (2016) Cost reduction of a hybrid energy storage system considering correlation between wind and PV power. Prot Control Mod Power Syst 1(1):11

[3] Li H, Abinet TE, Zhang JH et al (2017) Optimal energy management for industrial microgrids with high-penetration renewables. Prot Control Mod Power Syst 2(1):12

[4] Su HI, Gamal AE (2013) Modeling and analysis of the role of energy storage for renewable integration: power balancing. IEEE Trans Power Syst 28(4):4109-4117

[5] Chen Q, Liu D, Lin J et al (2015) Business models and market mechanisms of energy internet (1). Power Syst Technol 11(39):3050-3056

[6] Goebel C, Callaway DS (2013) Using ICT-controlled plug-in electric vehicles to supply grid regulation in California at different renewable integration levels. IEEE Trans Smart Grid 4(2):729-740

[7] Dallinger D, Krampe D, Wietschel M (2011) Vehicle-to-grid regulation reserves based on a dynamic simulation of mobility behavior. IEEE Trans Smart Grid 2(2):302-313

[8] Vatanparvar K, Al Faruque MA (2015) Design space exploration for the profitability of a rule-based aggregator business model within a residential microgrid. IEEE Trans Smart Grid 6(3):1167-1175

[9] Vagropoulos SI, Bakirtzis AG (2013) Optimal bidding strategy for electric vehicle aggregators in electricity markets. IEEE Trans Power Syst 28(4):4031-4041

[10] Short description of this project. https://cleanenergysolutions. org/sites/default/files/documents/pres-REFLEXE-gen-ext-EN201310-V2Yves.pdf. Accessed 26 Sep 2016

[11] Siebert N, Ammari S, Cao X et al (2015) Scheduling demand response and smart battery flexibility in a market environment: results from the reflexe demonstrator project. In: Proceedings of IEEE Eindhoven PowerTech, Eindhoven, Netherlands, 1-6 June 2015, pp 1-6

[12] Faghih A, Roozbehani M, Dahleh MA (2013) On the value and price-responsiveness of ramp-constrained storage. Energy Convers Manag 76:472-482

[13] Apt J, Walawalkar R, Mancini R (2007) Economics of electric energy storage for energy arbitrage and regulation in New York. Energy Policy 35(4):2558-2568

[14] Tan X, Wu Y, Tsang DHK (2016) Pareto optimal operation of distributed battery energy storage systems for energy arbitrage under dynamic pricing. Trans Parallel Distrib Syst 27(7):2103-2115

[15] Ekman CK, Jensen SH (2010) Prospects for large scale electricity storage in Denmark. Energy Convers Manag 51(6):1140-1147

[16] Shcherbakova A, Kleit A, Cho J (2014) The value of energy storage in South Korea's electricity market: a Hotelling approach. Appl Energy 125:93-102

[17] Kintner-Meyer M (2014) Regulatory policy and markets for energy storage in North America. Proc IEEE 102(7):1065-1072

[18] Cho J, Kleit AN (2015) Energy storage systems in energy and ancillary markets: a backwards induction approach. Appl Energy 147:176-183

[19] He G, Chen Q, Kang C et al (2015) Optimal bidding strategy of battery storage in power markets considering performancebased regulation and battery cycle life. IEEE Trans Smart Grid 7(5):2359-2367

[20] Koller M, Borsche T, Ulbig A et al (2013) Defining a degradation cost function for optimal control of a battery energy 
storage system. In: Proceedings of IEEE Grenoble PowerTech conference, Grenoble, France, 16-20 June 2013, pp 1-6

[21] Ortega-Vazquez MA (2014) Optimal scheduling of electric vehicle charging and vehicle-to-grid services at household level including battery degradation and price uncertainty. IET Gener Transm Distrib 8(6):1007-1016

[22] Arghandeh R, Woyak J, Onen A et al (2014) Economic optimal operation of community energy storage systems in competitive energy markets. Appl Energy 135:71-80

[23] Swierczynski M, Stroe DI, Stan AI et al (2014) Selection and performance-degradation modeling of $\mathrm{LiMO}_{2} / \mathrm{Li}_{4} \mathrm{Ti}_{5} \mathrm{O}_{12}$ and $\mathrm{LiFePO}_{4}$ battery cells as suitable energy storage systems for grid integration with wind power plants: an example for the primary frequency regulation service. IEEE Trans Sustain Energy 5(1):90-101

[24] Wang J, Purewal J, Liu P et al (2014) Degradation of Lithiumion batteries employing graphite negatives and nickel-cobaltmanganese oxide + spinel manganese oxide positives: part 1, aging mechanisms and life estimation. J Power Sour 269:937-948

[25] Dragičević T, Pandžić H, Škrlec D et al (2014) Capacity optimization of renewable energy sources and battery storage in an autonomous telecommunication facility. IEEE Trans Sustain Energy 5(4):1367-1378

[26] Offical website of Gams. http://www.gams.com/. Accessed 12 June 2016

[27] Short introduction for AMP20 Lithium-ion Prismatic Cell http:// www.a123systems.com/prismatic-cell-amp20.htm. Accessed 20 June 2016

[28] Schoenung SM, Hassenzahl WV (2003) Long-vs. short-term energy storage technologies analysis - a life-cycle cost study-a study for the DOE energy storage systems program. Sandia National Laboratories, Albuquerque. SAND2003-2783

Kangning LIU received the B.E. degree from the Department of Electrical Engineering at Tsinghua University, Beijing, China. His research interests include energy storage and power markets.

Qixin CHEN received the Ph.D. degree from the Department of Electrical Engineering at Tsinghua University, Beijing, China, in 2010. He is currently an Associate Professor at Tsinghua University. His research interests include low-carbon electricity, power system economics and optimization, power markets and power generation expansion planning.

Chongqing KANG received his Ph.D. degree from the Department of Electrical Engineering at Tsinghua University in 1997. He is now a Professor at Tsinghua University. His research interests include lowcarbon electricity, power system planning, power system reliability and load forecasting.

Wei SU is an engineer in Guangdong Electric Power Research Institute, his research interests include energy storage and electrochemistry.

Guobin ZHONG is an engineer in Guangdong Electric Power Research Institute, his research interests include energy storage and electrochemistry. 